\title{
Perceptually Lossless Video Compression with Error Concealment
}

\author{
Chiman Kwan \\ Signal Processing, Inc., USA \\ chiman.kwan@signalpro.net
}

\author{
Eric Shang \\ Applied Research LLC, USA \\ eric.l.shang@gmail.com
}

\author{
Trac D. Tran \\ Johns Hopkins University, USA \\ trac@jhu.edu
}

\begin{abstract}
We present a video compression framework that has several components. First, we aim at achieving perceptually lossless compression. Several well-known video codecs in the literature have been evaluated and the performance was assessed using several well-known performance metrics. Second, we investigated the impact of error concealment algorithms for handling corrupted pixels due to transmission errors in communication channels. Extensive experiments using actual videos have been performed to demonstrate the proposed framework.
\end{abstract}

\section{CCS Concepts}

- Computing methodologies Image processing

\section{Keywords}

Video Compression; Error Concealment; Perceptually Lossless.

\section{INTRODUCTION}

Video compression has been widely used in many commercial and military applications [8,29]. Well-known video codecs include X264 [14] and X265 [11]. In some applications such as security monitoring videos where video quality is of prime importance, people are still using lossless image compression algorithms such as JPEG [31] and JPEG-2000 [27] for compressing videos frame by frame. Some recent algorithms such as X264 [14] and X265 [11] also provide lossless compression options. Such a practice seems to be too conservative, as lossless compression can only achieve two to three times of compression.

JPEG, X264, and X265 are discrete cosine transform (DCT) based algorithms and JPEG-2000 is wavelet based. About 15 years ago, there were some development in DCT based algorithms where overlapped blocks known as lapped transforms (LT) were used to further improve the compression [5]. In the past few years, a group of researchers have incorporated LT [5] into an open source video codec known as Daala [6].

Permission to make digital or hard copies of all or part of this work for personal or classroom use is granted without fee provided that copies are not made or distributed for profit or commercial advantage and that copies bear this notice and the full citation on the first page. Copyrights for components of this work owned by others than the author(s) must be honored. Abstracting with credit is permitted. To copy otherwise, or republish, to post on servers or to redistribute to lists, requires prior specific permission and/or a fee. Request permissions from Permissions@acm.org.

ICVISP 2018, August 27-29, 2018, Las Vegas, NV, USA

(C) 2018 Copyright is held by the owner/author(s). Publication rights

licensed to ACM.

ACM 978-1-4503-6529-1/18/08 ..\$15.00

https://doi.org/10.1145/3271553.3271622
In this research, we aim at achieving perceptually lossless compression with 8:1 compression ratio for videos. We propose to apply several state-of-the-art video codecs (X264, X265 and Daala) to compress videos. Four performance metrics were used in our study. Moreover, since some applications involve the use of communication channels to transmit compressed videos back to a control center, there are corrupted pixels due to channel errors. We also investigate the possibility of using error concealment techniques to repair the corrupted pixels.

The key contribution of our project is to integrate three components (compression, error concealment, and perceptually lossless evaluation) in the video compression system into a single framework. Our paper is organized as follows. Section 2 summarizes the technical approach and its components. Section 3 summarizes all the experiments using actual images that are of interest to our customer. Finally, concluding remarks will be given in Section 4.

\section{TECHNICAL APPROACH}

\subsection{Proposed Video Compression and Error Recovery Framework}

In this research, we will perform objective evaluations of different compression algorithms in the market. This is to ensure that we will deliver the best approach to our customer. Our overall technical approach can be summarized as follows. First, we will survey the literature to determine the compression algorithms available in the market. At the same time, we will survey different performance metrics for algorithm evaluation. The focus will be on metrics that can better model human perception. We will also survey the error resilient algorithms and error concealment techniques. Second, we will obtain realistic maritime and sonar images for algorithm evaluation. Third, we will apply the various compression algorithms to the collected images and generate various performance metrics. Finally, we will also apply advanced algorithms to deal with the corrupted pixels due to channel errors.

Our proposed approach can be summarized in Figure 1.

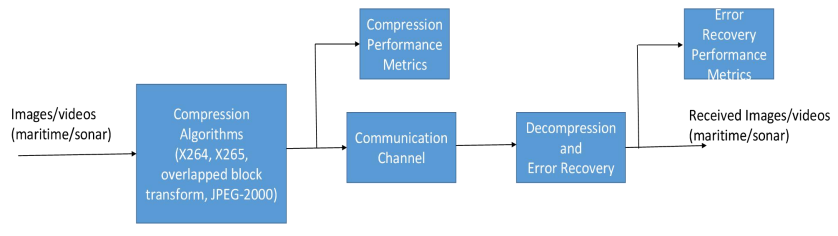

Figure 1. Proposed approach to evaluating different compression and error concealment algorithms.

\subsection{Survey of Compression Algorithms}

Instead of reinventing the wheels, we will compare image codecs in the market and objectively evaluate different codecs and eventually recommend the best codec to our customer. 
With the above in mind, we performed a survey of the existing high performance codecs, performance metrics, and error resilient coding.

\section{DCT based algorithms}

- JPEG [29]:

JPEG is the very first image compression standard. The video counterparts are the MPEG-1 and MPEG-2 standards.

- X-264 [14]:

$\mathrm{X} 264$ is the current state-of-the-art in video compression. Youtube uses X264. It has good still image compression.

- X-265[11]:

This is the next-generation video codec and has excellent still image compression and video compression. However, the computational complexity is much more than that of X264. In general, X265 has the same basic structure as previous standards. However, X265 contains many incremental improvement over X264:

$>$ More flexible partitioning, from large to small partition sizes

$>$ Greater flexibility in prediction modes and transform block sizes

$>$ More sophisticated interpolation and deblocking filters

$>$ More sophisticated prediction and signaling of modes and motion vectors

Features to support efficient parallel processing.

Several studies concluded that X265 yields the same quality as X264, but with only half of the bitrate. It should be noted that X264 and X265 are optimized versions of H264 and H265, respectively.

\section{- Daala [6]}

Recently, there is a parallel activity at xiph.org foundation, which implements a compression codec called Daala [6]. It is based on DCT. There are pre- and post-filters to increase energy compaction and remove block artifacts. Daala borrows ideas from [5], which was written by one of us (T. Tran). We call the above approach the lapped transform (LT) approach. In this study, we mainly used Daala in our experiments. We have mainly compressed images/videos using LT, X264, X265, and JPEG-2000.

- $\quad$ VP8 and VP9 [23,26]:

These video compression algorithms are owned by Google. The performance is somewhat close to X-264. However, it is not as popular as X264.

\subsection{Error Resilient/Error Concealment}

Although arithmetic coding achieves very high compression ratio, it is very vulnerable to channel errors. In [13], several coding schemes were evaluated. From [13], one can see that there is a limit to which the channel errors can be corrected. We propose to apply a combination of error resilient coding with error concealment. Our team has done extensive work in error concealment for Lidar, color, and hyperspectral images before.

\subsection{Principle of Video Error Concealment [2]}

Similar to still images, error resilient coding incurs more overhead in bandwidth and may not be able to deal with severe channel interferences. It is therefore necessary to apply error concealment techniques to recover lost pixels in the video.

As shown in Figure 2, our general approach for these problems is based on the observation that for every small block in a frame, there always exist some motion estimations (ME) from the previous or future frames. Taking into account both the temporal and spatial correlation, each patch with missing or corrupted values is grouped with similar patches according to block matching criteria from the partial information of the patch and stacked into columns of a matrix. All the matched motion estimations should have similar underlying image structures and the completed version of these patches should lie in a low dimensional subspace. Therefore, the constructed matrix becomes a very low-rank structure and low-rank techniques $[1,3,4,7,9,10,12,15,17,18,19,21,24,28,30]$ can be applied for reconstruction.

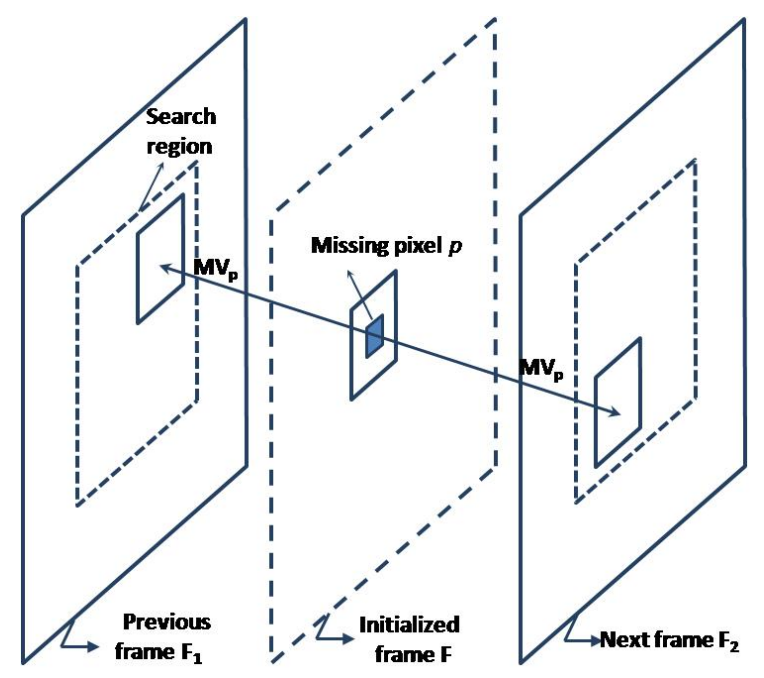

Figure 2. Bi-directional motion compensation approach to reconstruct corrupted pixels [2].

\subsection{Performance Metrics}

In almost all compression systems, researchers used peak signalto-noise ratio (PSNR) or structural similarity (SSIM) to evaluate the compression algorithms. Given a fixed compression ratio, algorithms that yield higher PSNR or SSIM will be regarded as better algorithms. However, PSNR or SSIM do not correlate well with human perception. Recently, a group of researchers investigated a number of different performance metrics [25]. Extensive experiments were performed to investigate the correlation between human perception with various performance metrics. According to the results found in [25], it was determined that two performance metrics correlate well with human perception. It was concluded that PSNR-HVS and PSNR-HVS-M have high correlation with human subjective evaluation results. Thus, we adopted these two metrics in our evaluations.

\section{EXPERIMENTAL RESULTS}

\subsection{Data}

We searched the internet and found some videos: one periscope video and one sonar video. The original frame rate was $30 \mathrm{fps}$. Here, we only show results for the periscope video due to page limitation. 


\subsection{Video Compression Results}

We resampled the videos to 6 fps. For 6 fps, we also focused only on the comparison between X264 and X265. Figure 3 to Figure 4 show that X264 performed better than X265 in all performance

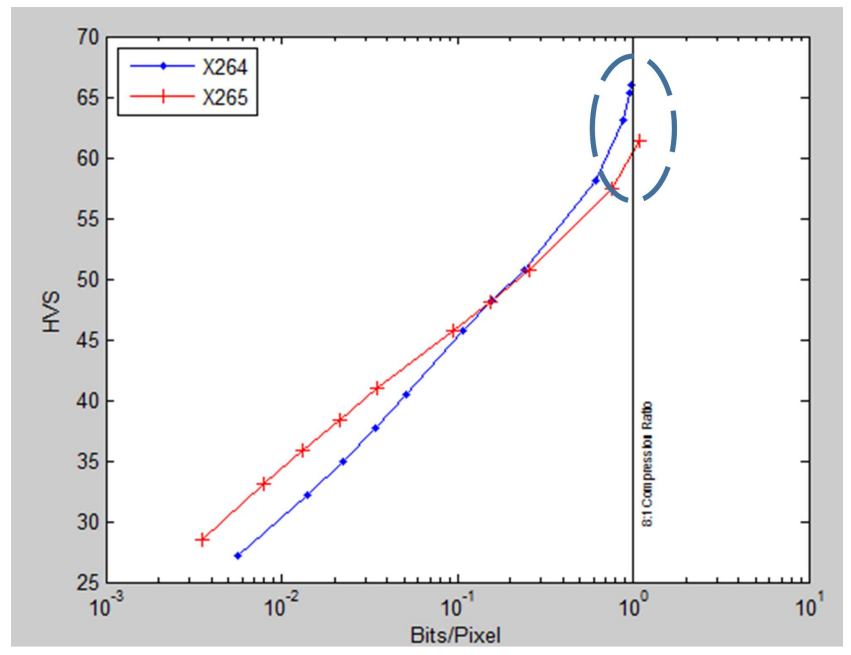

Figure 3. HVS exceeded $50 \mathrm{~dB}$ at 8:1.

\subsection{Video Error Concealment Results}

As mentioned in the literature survey section, error resilient coding can only protect the compressed bit stream to certain extent. If the interference in the communication channel is very severe due to either natural causes or enemy's intentional jamming, the bit stream will be heavily corrupted as a result. Hence, it is essential and absolutely necessary to have some high performance error concealment algorithms to repair the damaged packets. It is worth to mention that error concealment does not incur any bandwidth as compared to error resilient coding. Of course, some computational resource is needed at the receiving station, which should not be a problem in practice.

In this section, we applied our video error concealment approach to maritime videos. The theory has been described in an earlier metrics. In general, higher frame rate videos have better compression performance, which is reasonable due to more correlation between neighboring frames.

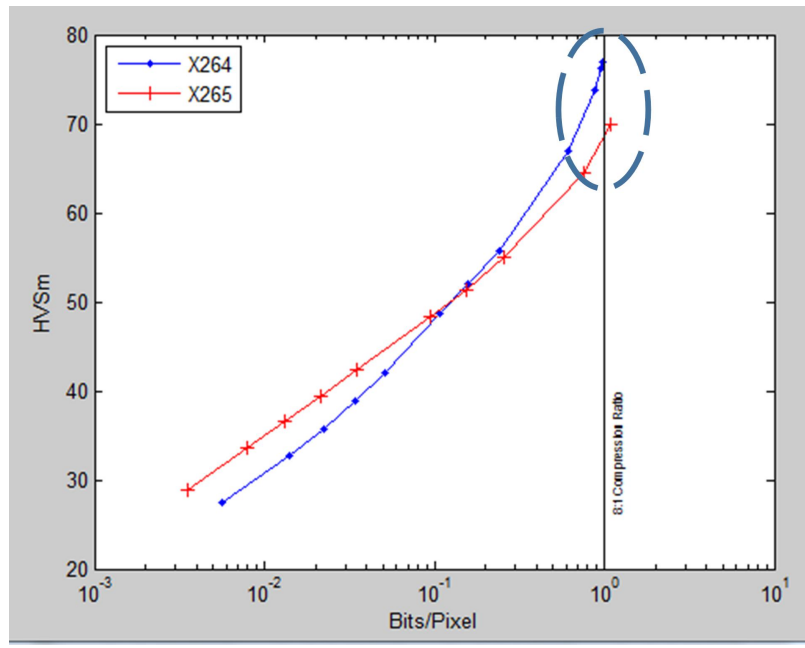

Figure 4. HVSm exceeded $70 \mathrm{~dB}$ at 8:1.

section. So we focus on presenting the results. The video size is $720 \times 1280$. Here we introduced a large corrupted block of size $400 \times 400$ on frame 6 . Figure 5 summarizes the original frame 6 , frame 6 with a large missing block due to corrupted packets during transmission, and a recovered frame 6 . For the $400 \times 400$ block, the PSNR is $36.48 \mathrm{~dB}$ for the reconstructed image.

Here, we compared our algorithm with another one called BMA (Boundary Matching Algorithm) [9]. It should also be noted that all frames have corrupted pixels. Figure 6 shows that our error recovery performance $(34.52 \mathrm{~dB})$ is $6.5 \mathrm{~dB}$ better than BMA $(28.02 \mathrm{~dB})$. This is very significant. One can also visually inspect the recovered images and notice that our performance is perceptually lossless.


Figure 5. Summary of error recovery in a periscope video. 

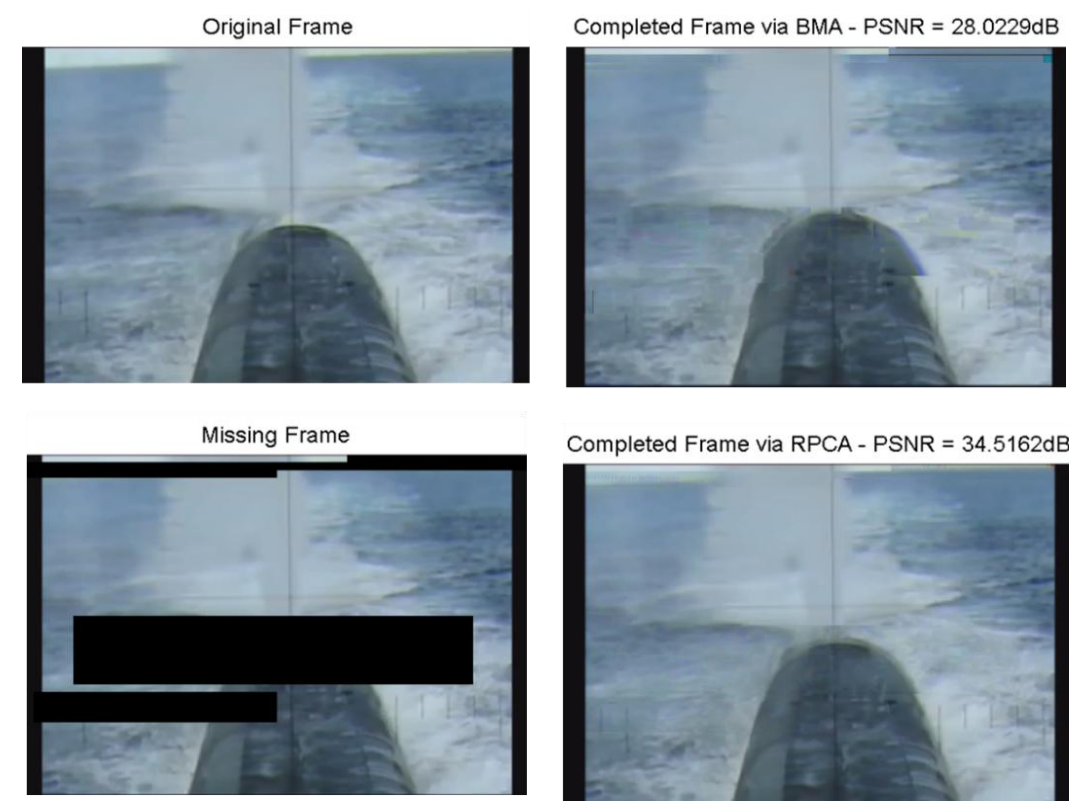

Figure 6. Video completion results for frame 5.

\section{CONCLUSIONS}

In this research, we have achieved perceptually lossless compression at 8:1 compression ratio for videos. The best video compression codec has been found via extensive experiments using four performance metrics. Moreover, we also applied an error concealment algorithm to repair those corrupted pixels due to transmission errors. We believe our algorithm can also be useful safe process and control monitoring applications [16][20].

\section{ACKNOWLEDGMENT}

This research was supported by NAVSEA under contract \# N141048-14-P-4560. This document has been approved for public release. Distribution is unlimited.

\section{REFERENCES}

[1] Chen, $Y$ et al. 2008 Video error concealment using spatiotemporal boundary matching and partial differential equation, IEEE Trans. on Multimedia, 10, p 2-15.

[2] Daala, http://xiph.org/daala/

[3] Dao, M, Kwan, C, Ayhan, B and Tran, T 2016 Burn Scar Detection Using Cloudy MODIS Images via Low-rank and Sparsity-based Models IEEE Global Conference on Signal and Information Processing, p $177-181$.

[4] Dogan, S, Sadka, A H and Kongoz, A M 2005 Error Resilient Techniques for Video Transmission Over Wireless Channels, Center for Communications System Research, U. Surrey.

[5] Dohner, J, Kwan, C and Ruggelbrugge, M 1996 Active Chatter Suppression in An Octahedral Hexapod Milling Machine: A Design Study, SPIE Smart materials \& Structure Conference, vol. 2721.

[6] Elad, M 2010 Sparse and Redundant Representations, Springer New York.

[7] JPEG, http://en.wikipedia.org/wiki/JPEG.

[8] JPEG-2000, http://en.wikipedia.org/wiki/JPEG_2000.
[9] Kwan, C and Luk, Y 2018 Hybrid sensor network data compression with error resiliency, Data Compression Conference.

[10] Kwan, C and Zhou, J 2015 Method for Image Denoising, Patent \#9,159,121.

[11] Kwan, C, Budavari, B, Dao, M and Zhou, J 2017 New Sparsity Based Pansharpening Algorithm for Hyperspectral Images IEEE Ubiquitous Computing, Electronics \& Mobile Communication Conference, $\mathrm{p}$ 88-93.

[12] Kwan, C, Li, B, Xu, R, Li, X, Tran, T and Nguyen, T Q 2001 Very Low-Bit-Rate Video Compression Using Wavelets," Wavelet Applications VIII, Proc. SPIE (vol. 4391), p 176180.

[13] Kwan, C, Shi, E and Um, Y 2018 High performance video codec with error concealment, Data Compression Conference.

[14] Kwan, C, Yin, J, Zhou, J, Chen, H and Ayhan, B and 2013 Fast Parallel Processing Tools for Future HyspIRI Data Processing, NASA HyspIRI Science Symposium.

[15] Nguyen, D, Dao, M and Tran, T D 2011 Error concealment via 3-mode tensor approximation, IEEE Int. Conf. on Image Processing.

[16] Pan, G, Xu, H, Kwan, C, Liang, C, Haynes, L S and Geng, Z 1996 Modeling and Intelligent Chatter Control Strategies for a Lathe Machine," Control Engineering Practice, 4, p 16471658.

[17] Ponomarenko, N, et al. 2007 On between-coefficient contrast masking of DCT basis functions Proc. of the Third International Workshop on Video Processing and Quality Metrics for Consumer Electronics.

[18] Strang G and Nguyen, T 1997 Wavelets and filter banks, Wellesley-Cambridge Press.

[19] Tran, T D, Liang, J and Tu, C 2003 Lapped transform via time-domain pre-and post-filtering IEEE Transactions on Signal Processing, 51, p 1557 - 1571. 
[20] VP8, http://en.wikipedia.org/wiki/VP8.

[21] VP9, http://en.wikipedia.org/wiki/VP9.

[22] Wang, W, Li, S, Qi, H, Ayhan, B, Kwan, C and Vance, S 2015 Identify Anomaly Component by Sparsity and Low Rank, IEEE Workshop on Hyperspectral Image and Signal Processing: Evolution in Remote Sensor (WHISPERS).

[23] Wu, J, Liang, Q and Kwan, C 2012 A Novel and Comprehensive Compressive Sensing based System for Data Compression," Proc. IEEE Globecom, Anaheim, CA.

[24] X264, http://www.videolan.org/developers/x264.html

[25] X265, https://www.videolan.org/developers/x265.html

[26] Zhou, J and Kwan, C 2018 A Hybrid Approach for Wind Tunnel Data Compression Data Compression Conference, Snowbird, Utah, March 27-30.

[27] Zhou, J and Kwan, C 2018 High Performance Image Completion using Sparsity based Algorithms, SPIE Commercial + Scientific Sensing and Imaging Conference.
[28] Zhou, J and Kwan, C, 2018 Missing Link Prediction in Social Networks, 15th International Symposium on Neural Networks.

[29] Zhou, J, Ayhan B, Kwan, C and Tran, T 2018 ATR Performance Improvement Using Images with Corrupted or Missing Pixels, SPIE Defense + Security Conference.

[30] Zhou, J, Kwan, C and Ayhan B 2012 A High Performance Missing Pixel Reconstruction Algorithm for Hyperspectral Images, 2nd. International Conference on Applied and Theoretical Information Systems Research.

[31] Zhou, J, Kwan, C and Ayhan B 2012 A High Performance Missing Pixel Reconstruction Algorithm for Hyperspectral Images, 2nd. International Conference on Applied and Theoretical Information Systems Research. 